\title{
Erratum to "Esophageal Pemphigus Vulgaris: A Rare Etiology of Upper Gastrointestinal Hemorrhage"
}

\author{
Jennifer Rose F. Del Castillo $\mathbb{D i D}^{1,2,3,4}$ Muhammad Nadeem Yousaf ${ }^{1}{ }^{1,2,3,4}$ \\ Fizah S. Chaudhary $\mathbb{D}^{1,},^{1,2,3,4}$ Nahar Saleh $\mathbb{D}^{1,},{ }^{1,2,3,4}$ and Lawrence Mills ${ }^{5}$ \\ ${ }^{1}$ Department of Medicine, MedStar Union Memorial Hospital, Baltimore, MD 21218, USA \\ ${ }^{2}$ MedStar Good Samaritan Hospital, Baltimore, MD 21239, USA \\ ${ }^{3}$ MedStar Franklin Square Medical Center, Baltimore, MD 21237, USA \\ ${ }^{4}$ MedStar Harbor Hospital, Baltimore, MD 21225, USA \\ ${ }^{5}$ Department of Gastroenterology, MedStar Good Samaritan Hospital, Baltimore, MD 21239, USA
}

Correspondence should be addressed to Muhammad Nadeem Yousaf; muhammad.n.yousaf@medstar.net

Received 8 May 2021; Accepted 8 May 2021; Published 29 May 2021

Copyright (C) 2021 Jennifer Rose F. Del Castillo et al. This is an open access article distributed under the Creative Commons Attribution License, which permits unrestricted use, distribution, and reproduction in any medium, provided the original work is properly cited.

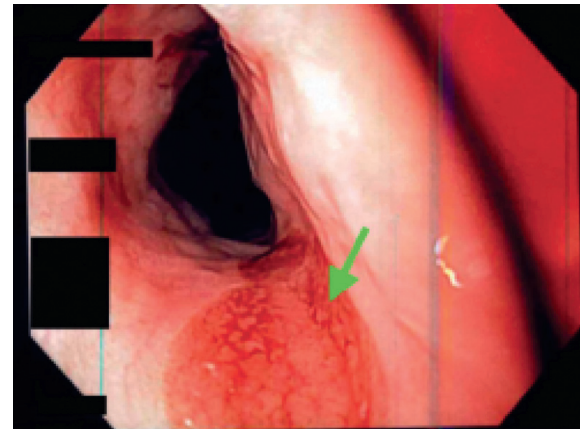

(a)

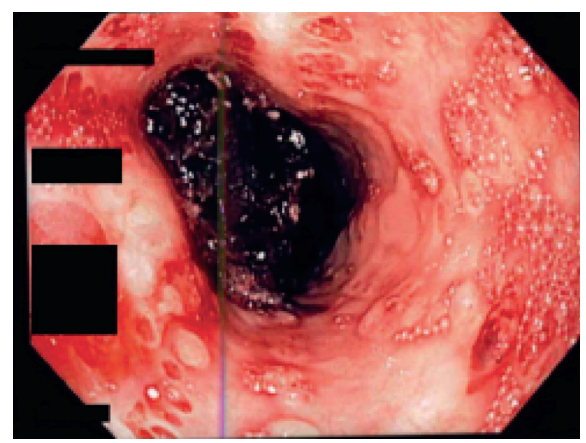

(c)

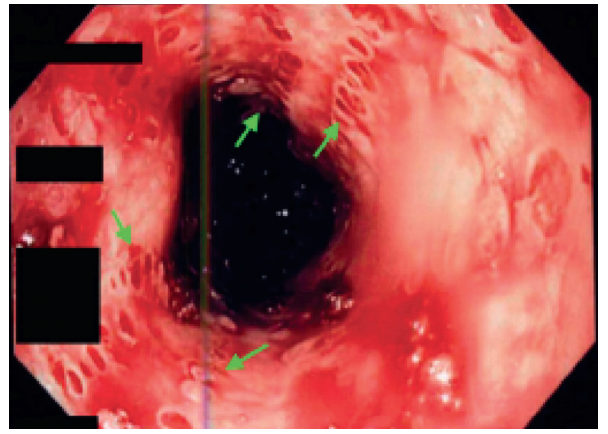

(b)

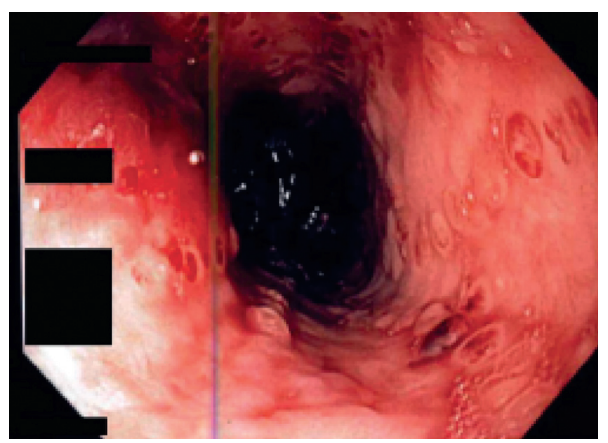

(d)

FiguRE 1: Upper endoscopic evaluation showing multiple mucosal ulcers with blisters throughout the esophagus suggestive of pemphigus vulgaris. 
In the article titled "Esophageal Pemphigus Vulgaris: A Rare Etiology of Upper Gastrointestinal Hemorrhage" [1], the published version of Figure 1 should be rotated in clockwise direction. This also includes the arrangement of the photos to indicate location within the GI tract. This mistake was introduced during the production of the article by the publisher, and we sincerely apologise for this. The corrected figure is shown below and is listed as Figure 1.

\section{References}

[1] J. R. F. Del Castillo, M. N. Yousaf, F. S. Chaudhary, N. Saleh, and L. Mills, "Esophageal pemphigus vulgaris: a rare etiology of upper gastrointestinal hemorrhage," Case Reports in Gastrointestinal Medicine, vol. 2021, Article ID 5555961, 5 pages, 2021. 